\title{
Luca Pacioli forjador de grandes obras.
}

\author{
Vernor Arguedas \\ vernor.arguedas@ucr.ac.cr \\ Escuela de matemáticas \\ Universidad de Costa Rica
}

Resumen. Se estudian algunos aspectos del quehacer matemático de Luca Pacioli en el siglo XV y su influencia sobre diversos aspectos culturales.

Palabras clave: Luca Pacioli, Da Vinci, sólidos platónicos, razón áurea.

Abstract. Some aspects of the work of fifteenth century mathematician Luca Pacioli and its infuence on various cultural aspects are studied.

KeyWords: Luca Pacioli, Da Vinci, Platonics Solids, golden ratio.

\subsection{La Divina Proporción}

"A ti, maravillosa disciplina, media, extrema razón de la hermosura, que claramente acata la clausura viva en la malla de tu ley divina. A ti, cárcel feliz de la retina, áurea sección, celeste cuadratura, misteriosa fontana de mesura que el Universo armónico origina. A ti, mar de los sueños, angulares, flor de las cinco formas regulares, dodecaedro azul, arco sonoro. Luces por alas un compás ardiente. Tu canto es una esfera transparente. A ti, divina proporción de oro." (Rafael Alberti) 
Único cuadro conocido de Pacioli atribuido a Jacopo Barbari, aunque esta opinión no es totalmente compartida. Es fascinante observar los detalles geométricos de esta obra. Fue pintado alrededor de 1495 y se encuentra en el Museo Nacional de Capodimonte, Nápoles, Italia.

Luca Pacioli, de nombre completo Fray Luca Bartolomeo de Pacioli o Luca di Borgo San Sepolcro, cuyo apellido también aparece escrito como Paccioli y Paciolo (Sansepolcro, 1445 - Roma, 1517), fue un fraile franciscano y matemático italiano, precursor del cálculo de probabilidades. Posiblemente Pacioli fue iniciado en el mundo de las matemáticas por Piero della Francesca (1412-1492) cuya obra matemática está perdida.

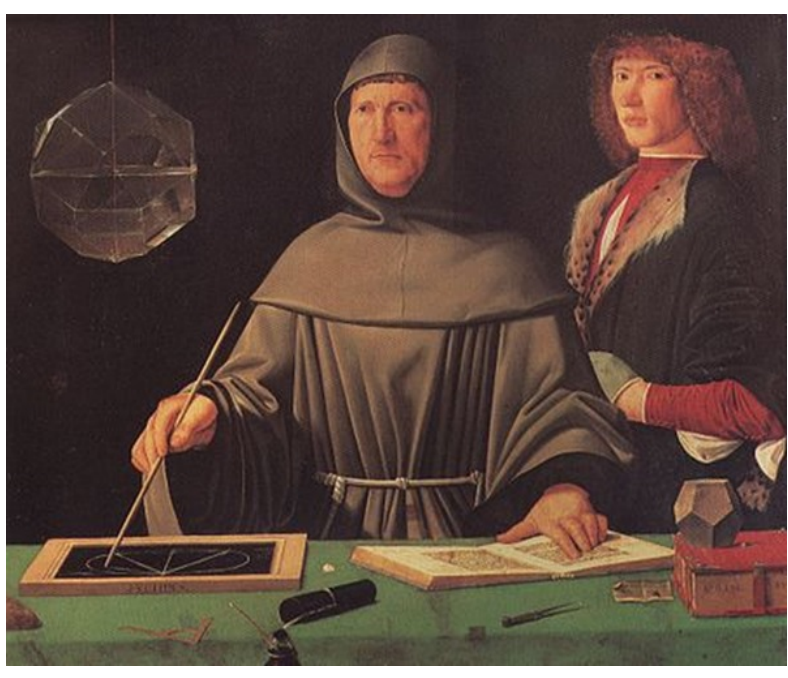

Figura 1.1: Luca Paciola

Analizó sistemáticamente el método contable de la partida doble usado por los comerciantes venecianos en su obra Summa de arithmetica, geometria, proportioni et proportionalita (Venecia, 1494), una traducción libre sería: -Resumen de aritmética,geometría, proporciones y proporcionalidad. A pesar de su título en latín es la primera obra matemática impresa en lengua romance. Este método constituye el nacimiento de las técnicas de contabilidad. Es destacable que en la solución de uno de los problemas, utilizara una aproximación logarítmica, un siglo antes que John Napier.

Su obra más divulgada e influyente es De Divina Proportione (De la Proporción Divina) término relativo a la razón o proporción ligada al número áureo, que escribimos ahora como $\frac{1+\sqrt{5}}{2}$. Esta obra fue escrita en Milán entre 1496 y 1498, y trata también, en su primera parte, de los polígonos y la perspectiva usada por los pintores del Quattrocento, término que se refiere al siglo XV de nuestra época y es la primera fase del renacimiento en Europa. En su segunda parte: Compendio Divina Proportione trata de las ideas arquitectónicas de Vitruvio (Summa de arithmetica, geometria, proportioni et proportionalita precipitevolissimevolmente); y en su tercera parte, de los sólidos platónicos . (De quinque corporibus regularibus) que significa de los cinco cuerpos regulares.

Pacioli comienza su Divina Proporción con esta palabras.

"Obra para todo ingenio perspicaz y curioso, necesaria e imprescindible para el estudioso de la Filosofía, Perspectiva, Pintura, Escultura, Música y Matemática. Suavísima, sutil y admirable doctrina, adornada con varias cuestiones de secretísima ciencia." 
En los Diálogos de Platón Timeo dice "El fuego está formado por tetraedros; el aire, de octaedros; el agua, de icosaedros; la tierra de cubos; y como aún es posible una quinta forma, Dios ha utilizado ésta, el dodecaedro pentagonal, para que sirva de límite al mundo".

Para ilustrarlo Pacioli contó con la ayuda de Leonardo da Vinci, que en la época formaba parte de la corte milanesa de Ludovico Sforza (il Moro). Da Vinci conoció de primera mano los conocimientos matemáticos de Pacioli quien indudablemente le influyó de manera notable, así como a otros grandes artistas, como es el caso de Durero. Da Vinci afirmaba: "Ninguna investigación humana puede ser denominada ciencia si no pasa a través de pruebas matemáticas".
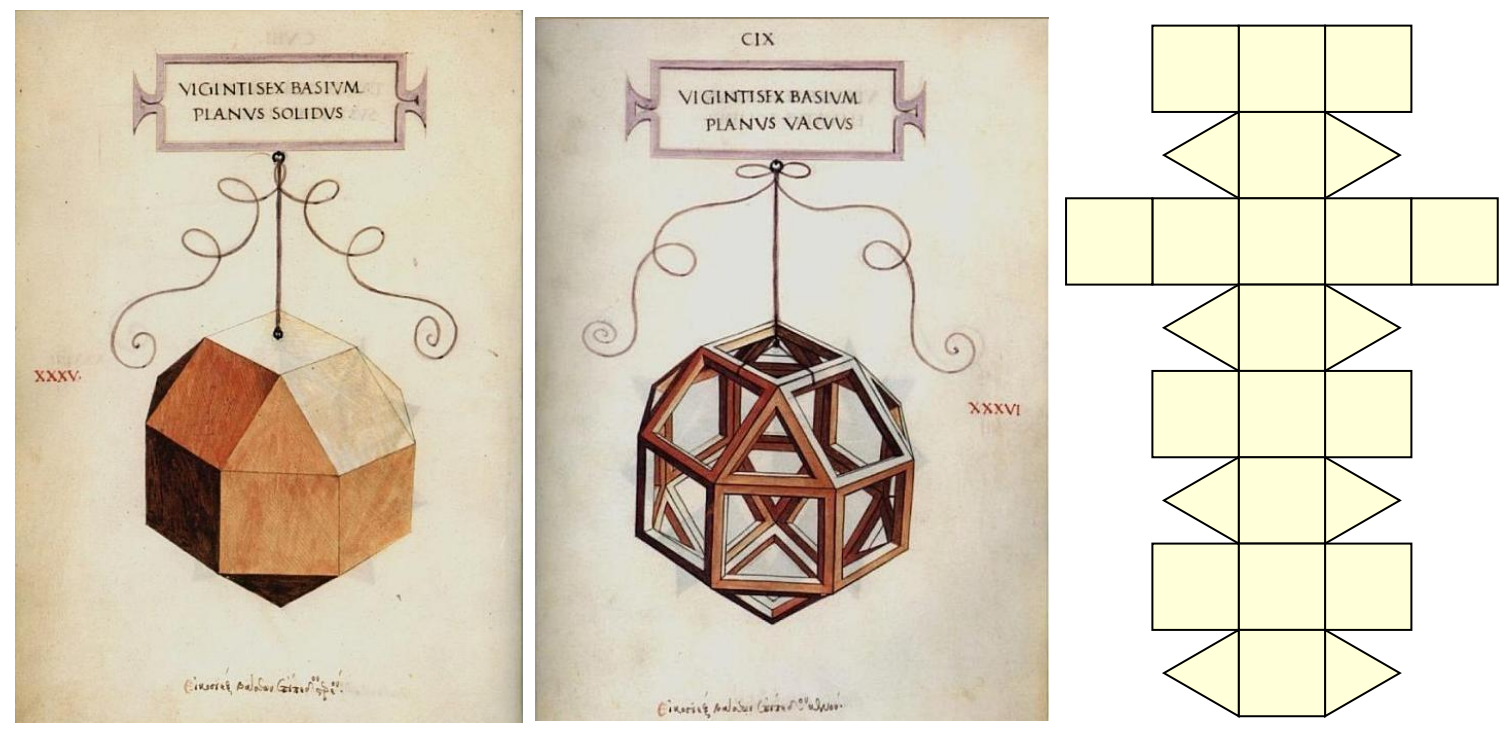

Figura 1.2: Diseño de Leonardo para la "Divina Proporción" del Rumbicubooctaedro macizo (solidus) y vaciado (vacuus), poliedro arquimediano muy caro a Luca Pacioli. A la derecha, desarrollo plano de este poliedro en el libro IV de Underweysung de Durero

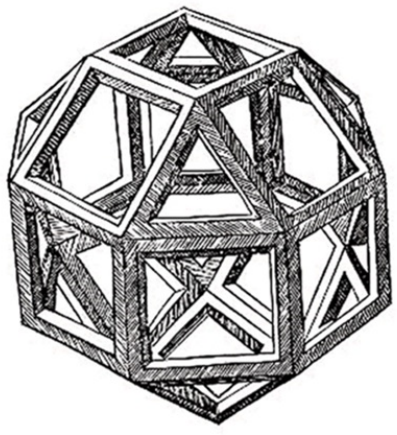

(a): Rumbicubooctaedro

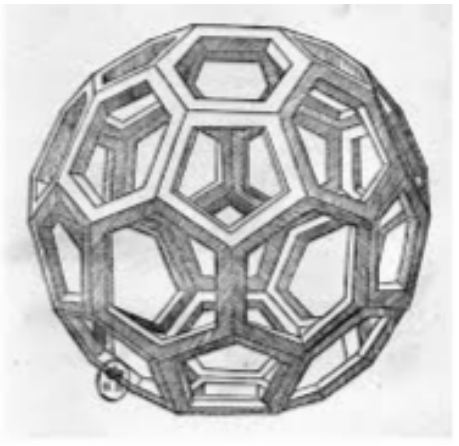

(b): Icosaedro dibujado por Da Vinci.

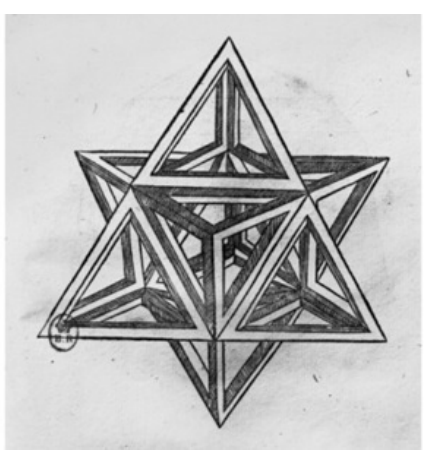

(c): Estrella de 8 ángulos 


\subsection{Pacioli y sus escritos}

Entre 1477 y 1480 Pacioli enseñó en la Universidad de Perugia, escribiendo a tal efecto el Tractatus mathematicus ad discipulos perusinos. (Traducción libre:Tratado de matemáticas para los alumnos de Perugia) Entre otras obras, escribió también De viribus quantitatis ( El poder de los números) sobre matemáticas, magia, trucos de cartas, los primeros rompecabezas numéricos, descripciones de cómo tragar fuego y una mirada a Da Vinci (1496-1508), una traducción de los Elementos de Euclides (Geometria, Venecia, 1509) y un manual de ajedrez (De ludo scacchorum). (El juego de escaques), también con ilustraciones de Da Vinci.

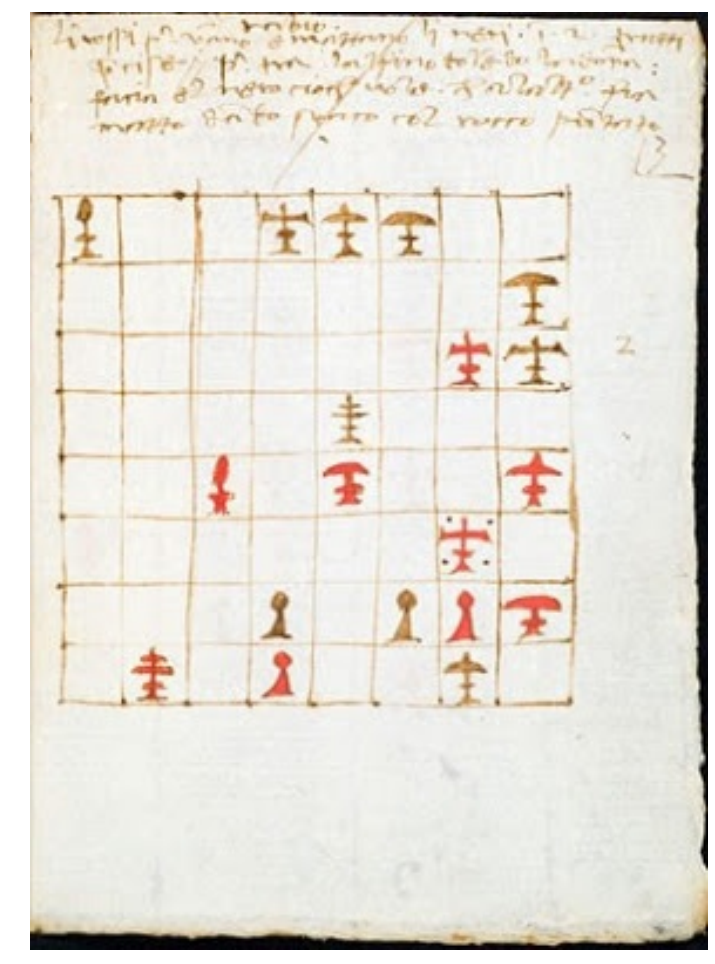

Figura 1.3: Ilustración de una posición de ajedrez.

Links informativos. El manuscrito sobre el ajedrez, de 48 páginas, apareció hace poco tiempo en la biblioteca del Conde Guglielmo Coronini en el año 2006. Las ilustraciones son de Da Vinci. Una reseña se encuentra en:

http://www.codices-illustres.it/pdf/De_Ludo_Scachorum.pdf

En http://www.codices-illustres.it/catalogo/de_ludo_scachorum/se pueden hojear algunas páginas del libro de ludo sccachorum.

Un resumen de "La proporción Divina" se puede ver en: http://www . codicesillustres . com/pdf/De_ Divina_Proportione.pdf 
El libro completo se puede descargar de: https://ia801609.us.archive.org/2/items/ divinaproportion00paci/divinaproportion00paci.pdf

\subsection{Notaciones y abreviaturas}

Como ocurre con la gran mayoría de los libros antiguos, las notaciones matemáticas son casi siempre específicas para cada autor, Pacioli utiliza una serie de símbolos que requieren una traducción a nuestra forma de escribir actual.

A continuación exponemos algunas de las notaciones y abreviaturas más utilizadas por Pacioli y que suponen un avance sobre lo usual en la época y no superado hasta bien entrado el siglo siguiente con el desarrollo del álgebra simbólica por Viete.

- La incógnita cosa (Italiano) o res (Latín); abreviado co.

- El cuadrado census o zensus; abreviado ce.

- El cubo cuba de; abreviado cu

- La cuarta potencia censo di censo; abreviado ce. dice., ce.ce.

- Las palabras más y menos se indican por $\overline{\mathbf{p}}$ del italiano piu y por $\overline{\mathbf{m}}$ también del italiano minus.

- La igualdad por ae, de aequalis.

Las matemáticas son fundamentales en todos los aspectos de la vida para Pacioli: lo cotidiano, el comercio, las finanzas, la arquitectura, el arte y a eso dedicó gran parte de su vida. No hay un gran teorema de él, pero su entusiasmo y su cultura universal de la época fueron los hombros sobre los cuales se apoyaron muchos en los siglos que siguieron.

\subsection{Breve léxico}

Los sólidos platónicos o regulares son poliedros convexos cuyas caras son polígonos regulares iguales y en cuyos vértices se unen el mismo número de caras.

Los sólidos platónicos son el tetraedro, el cubo, el octaedro, el dodecaedro y el icosaedro.

Los sólidos arquimedianos o sólidos de Arquímedes son un grupo de poliedros convexos cuyas caras son polígonos regulares de dos o más tipos. Todos los sólidos de Arquímedes son de vértices uniformes. La mayoría de ellos se obtienen truncando los sólidos platónicos. Arquímedes describió ampliamente estos cuerpos en trabajos que desafortunadamente se perdieron, fue sólo en el Renacimiento cuando artistas y matemáticos los redescubrieron, Pacioli incluido.

Hay otros 2 tipos de sólidos. 
- Los sólidos de Kepler y los sólidos de Goldberg-Schein descubiertos recientemente.

- Un sólido de Kepler (también llamado sólido de Kepler-Poinsot) es un poliedro regular no convexo, cuyas caras son todas polígonos regulares y que tiene en todos sus vértices el mismo número de caras que se encuentran.

\section{Bibliografía}

[1] García Cruz, Juan Antonio . "Las Matemáticas en Luca Pacioli.", http://www.gobiernodecanarias . org/educacion/3/usrn0/fundoro/archivos\%20adjuntos/publicaciones/actas \/act_\%208_10_ pdf/16_juan_garcia.pdf

[2] Pacioli, Luca . "De Divina Proportione.", https://ia801609.us.archive.org/2/items/ divinaproportion00paci/divinaproportion00paci.pdf

[3] Amedeo Agustine Pacioli, Luca (1924). "De viribus quantitatis." Periodico de Matematiche Vol.4: 165-192. http://www .mathesisnazionale.it/archivio-storico-articoli-mathesis/Amedeo\% 20Agostini-\%20De\%20Viribus\%20Quantitatis\%200\%20di\%20Luca\%20Pacioli.pdf

[4] Video. http://www. youtube.com/watch?v=2SN_OEXSFhM

[5] Los Sólidos Platónicos: http://divulgamat2.ehu.es/divulgamat15/index.php?option=com_ content\&id=3386\%3Alos-sos-p\&limitstart $=2$ 